\title{
La informalidad y su incidencia en la evasión tributaria de los comerciantes de abarrotes de la localidad de Balsahuayco - Jaén, primer semestre 2021
}

\author{
Ramos Rodriguez, Yecenia Yanilet \\ Ramos.yesi22@gmail.com \\ https://orcid.org/0000-0002-3491-9942 \\ Consorcio Universidad César Vallejo \\ Moyobamba - Perú.
}

\section{RESUMEN}

El presente trabajo de investigación tiene como objetivo principal determinar la incidencia de la Informalidad en la Evasión Tributaria de los comerciantes de abarrotes de la localidad de Balsahuayco - Jaén, primer semestre 2021. Investigación de tipo básica, con diseño no experimental de corte transversal, con una muestra de 40 comerciantes de abarrotes, a quienes se les aplicó la encuesta, obteniendo como resultado que el 100.0\% de los comerciantes de abarrotes de la localidad de Balsahuayco son informales, ocasionando así las evasiones tributarias en dicha localidad, determinándose que la informalidad incide de manera negativa en la evasión tributaria, demostrado mediante una correlación Rho de Spearman de 0,855 y un nivel de significancia de 0,01.. Es decir, que el desconocimiento sobre el proceso de formalización, la ausencia de compromiso social y los factores de informalidad inciden en las deficiencias de la recaudación tributaria, la fiscalización y el incumplimiento tributario, generando así que el país no logre su tan anhelado crecimiento.

Palabras clave: Informalidad; Evasión tributaria; Fiscalización tributaria 


\title{
Informality and its impact on tax evasion of grocery merchants in the town of Balsahuayco - Jaén, first semester 2021
}

\begin{abstract}
The main objective of this research work is to determine the incidence of Informality in Tax Evasion of grocery merchants in the town of Balsahuayco - Jaén, first semester 2021. Basic type research, with a non-experimental cross-sectional design, with a sample of 40 grocery merchants, to whom the survey was applied, obtaining as a result that $100.0 \%$ of the grocery merchants in the town of Balsahuayco are informal, thus causing tax evasion in said locality, determining that informality affects negatively in tax evasion, demonstrated by a Spearman's Rho correlation of 0.855 and a significance level of 0.01 . That is, ignorance about the formalization process, lack of social commitment and informality factors affect deficiencies in tax collection, auditing and tax non-compliance, thus generating that the country does not achieve its long-awaited growth.
\end{abstract}

Keywords: Informality; Tax evasion; Tax inspection.

Artículo recibido: 30 noviembre. 2021 Aceptado para publicación: 29 diciembre 2021 Correspondencia: cristina.chamorro@espoch.edu.ec Conflictos de Interés: Ninguna que declarar 


\section{INTRODUCCIÓN}

Internacionalmente en los últimos años, según Chávez, Peña, \& Velazco (2015), el sector informal va en crecimiento en países desarrollados y en países subdesarrollados situación que obliga a poner cuidado al tamaño, estructura y evolución de este sector. Hernández \& De la Roca (2016) han podido identificar hasta cuatro motivos principales que causan la informalidad e diversos países: En primer lugar se tiene que las acciones informales tienen menos base impositiva y con ellos se impide financiar los bienes públicos y de protección social; el segundo es la distorsión estadística que causan las actividades informales prosperas, con lo que los fallos de política establecidas en las guías, suelen ser insuficientes o inversas a los objetivos deseados; el tercero es que una economía semejante en cúspide puede cautivar mano de obra y avivar una competencia desigual con las entidades formales; y por último la sección informal, puede generar spillovers positivo para la economía, debido a que fija un ánimo corporativo y dispuesto, aumentando la competencia y por ende su nivel de eficiencia.

En el Perú, Verona (2019) señala que la principal fuente de evasión fiscal se da en la parte informal de la economía, en INEI encontramos que más del $80 \%$ de las empresas nacionales son informales. Para Luján \& Cano (2019), en el país tenemos muchos impuestos y regulaciones para las actividades de producción y en actividades generalmente de ingresos, y efectuar en un estudio sobre su cumplimiento o no por parte de los individuos y organizaciones con respeto al marco institucional o si el cumplimiento es parcial en situaciones que ocurren realmente y entonces en el estudio sobre las dimensiones de la informalidad no solamente debe considerarse las actividades que naturalmente escapan del registro oficial y de la ley, debe tenerse en cuenta también a las evasiones que se dan en ingresos resultantes de actividades que se rigen a la ley aparentando ser formales.

Balsahuayco es una localidad ubicada en el distrito de Jaén, provincia de Jaén, Región de Cajamarca. En dicha localidad la mayoría de los Comerciantes de Abarrotes son informales por eso se da la evasión tributaria. La evasión tributaria, afecta el recaudo de impuestos, por ello se ha convertido en un inconveniente para la Municipalidad de la Provincia de Jaén porque la recaudación es mínima, esto conlleva a la insolvencia de proyectos que cubran las necesidades de las zonas rurales y urbanas. El problema más elevado de evasiones es por la informalidad del sector Comercio, lo que garantiza además 
el incumplimiento de las metas y objetivos planteados por el MEF. (Alcalde Huanca, 2019).

La justificación teórica se da, teniendo en cuenta los conceptos dados por autores, como Quispe (2020), quien menciona que la informalidad se da en entidades, recursos humanos y acciones que trabajan sin tener en cuenta la ley que manda en la actividad económica; además, Collosa (2019), nos dice que la evasión es reducir impuestos utilizando medios ilegales. La justificación práctica se da al despertar inquietudes sobre el uso de aplicar buenas costumbres en contribuyentes que quieran realizar buenas prácticas tributarias, también se da por el Gobierno, Entidades Públicas y la superintendencia nacional de aduanas y administración tributaria. Lo que lleva a la SUNAT a ser más competente, e impulsa a llevar un mejor control en la participación de los funcionario y entidades. La justificación por conveniencia se da porque ayudará a todas las empresas con la misma problemática en la informalidad de la localidad de Balsahuayco, lo que ayudaría con conocimientos acerca del problema que causa la informalidad a la economía del país, y responder las cuestiones en cuanto a las evasiones tributarias. Por último, la justificación social se da al facilitar información precisa que influya de manera positiva en la toma de decisiones, para que la informalidad vaya desapareciendo en el sector de comercio de abarrotes del Centro Poblado Balsahuayco, fortaleciendo a los empleos de manera formal, acceso a financiamiento seguro, mejorar la calidad de los productos y servicios y mantener la estabilidad económica y el desarrollo social.

Por ello la presente investigación busca responder al problema general: ¿De qué manera incide en la Informalidad en la Evasión Tributaria de los comerciantes de abarrotes de la localidad de Balsahuayco - Jaén, primer semestre 2021? Y los problemas específicos: ¿Cómo el desconocimiento sobre formalización incide en la Evasión Tributaria de los comerciantes de abarrotes de la localidad de Balsahuayco - Jaén, primer semestre 2021?, ¿Cómo la ausencia de compromiso social incide en la Evasión Tributaria de los comerciantes de abarrotes de la localidad de Balsahuayco - Jaén, primer semestre 2021 ? Y ¿Cómo los factores de informalidad inciden en la Evasión Tributaria de los comerciantes de abarrotes de la localidad de Balsahuayco - Jaén, primer semestre 2021 ? Para lo que se tiene la Hipótesis General: La informalidad incide de manera negativa en la Evasión Tributaria de los comerciantes de abarrotes de la localidad de Balsahuayco Jaén, primer semestre 2021. 
Teniendo como objetivo general: Determinar la incidencia de la Informalidad en la Evasión Tributaria de los comerciantes de abarrotes de la localidad de Balsahuayco Jaén, primer semestre 2021; y objetivos específicos: Determinar la incidencia del desconocimiento sobre formalización en la Evasión Tributaria de los comerciantes de abarrotes de la localidad de Balsahuayco - Jaén, primer semestre 2021, Determinar la incidencia de la ausencia de compromiso social en la Evasión Tributaria de los comerciantes de abarrotes de la localidad de Balsahuayco - Jaén, primer semestre 2021 y Determinar la incidencia de los factores de informalidad en la Evasión Tributaria de los comerciantes de abarrotes de la localidad de Balsahuayco - Jaén, primer semestre 2021.

\subsection{Trabajos previos}

Como antecedentes internacionales tenemos a: Bu \& Cuervo (2020). "Informality costs: Informal entrepreneurship and innovation in emerging economies". Artículo Científico que concluye que los costos de informalidad quedan impresos y afectan las relaciones de agencia internas entre empleados y gerentes y las relaciones de agencia externa con proveedores y distribuidores, limitando los incentivos de las empresas y su capacidad para innovar incluso después de la formalización. Como resultado, las empresas creadas informalmente se involucran más en la imitación y menos en el desarrollo de nuevos productos innovadores. Ulyssea (2020). "Informality: Causes and Consequences for Development”. Artículo científico que concluye que la reducción de los costos de la formalidad no es una política eficaz para reducir la informalidad, pero puede generar efectos agregados positivos, como una mayor producción y productividad total de los factores. Lo mejor es aumentar la aplicación en el margen extensivo, pero no en el margen intensivo de la informalidad. Paredes (2017). "La evasión tributaria y la recaudación generada por los comerciantes informales de ropa del mercado mayorista de la ciudad de Ambato". Tesis que concluye que la evasión tributaria se genera por la ineficiente redistribución de lo recaudado, las suspensiones socio económico en los comercios, es por ello que este sector se encuentra desinformado y no existe un cumplimiento a gran escala.

En los nacionales tenemos a Quispe (2016). "La informalidad y su relación con la evasión tributaria en los negociantes del distrito de Anco Huallo 2016". Tesis que concluye que existen tres factores que aumentan la informalidad; los impuestos por trabajadores, pésimo marco legal en seguridad nacional, políticas económicas que inquietan a las 
secciones más prósperos en elegir la informalidad y reformas comerciales que están hechas sin tener el impacto que podrían causar en las secciones de menor productividad. Fossa (2017). “Análisis de la informalidad y evasión tributaria en los hospedajes ubicados en la urbanización Piura 2017'. Tesis que concluye que la mayoría de los hospedajes son informales porque no saben el proceso de la formalización, ósea no conocen los trámites, licencias y otros documentos para empezar con buen pie los negocios, además, no tienen compromiso para el cumplimiento del pago de tributos, debido a que no conocen sus derechos. y el fin de financiar al estado con la tributación. Cubas (2017). "Consecuencia de la informalidad de las MYPES en los recaudos tributarios en el distrito de José Leonardo Ortiz de la provincia de Chiclayo año 2016-2017“. Tesis que llega a la conclusión que la insensibilización de las jurisdicciones y la terquedad de las MYPES informales crean discrepancia en ambos fragmentos por lo que siempre habrá un desacuerdo recíproco, así las acciones informales de las MYPES tendrán un efecto perjudicial en el cobro de tributos.

\subsection{Teorías relacionadas al tema}

La informalidad dependiendo de la realidad que vive cada país adopta diversas, para Adriano (2018), en Perú se puede definir como informal a los negocios, empresas que no van acorde el marco constitucional de la ley de sociedades y quienes no contratan personal dentro de las leyes laborales. Según Rodríguez \& Dussán (2018), cuando son informales las entidades quedan limitadas para su desarrollo normal porque no tiene entrada al Sistema Financiero, tiene más gastos por cubrir sus actividades, no tiene beneficios legales, no aprovecha las economías de escala, etc. Esto se deriva en la incapacidad de difundir su mercado interno y externo. Esos elementos prejudiciales se convierten en productividad mínima, formando una disminución de competitividad y debido a ello no crece la economía. (Dienes, 2018).

Lavado (2016), señala que la informalidad hace parte para que un país sea subdesarrollado, esto se da por el tipo de distribución socioeconómica que son heredadas por economías en transformación hacia la renovación como se da por la analogía que instituye el gobierno con los empleados privados por medio de la regulación, el monitoreo y la provisión de servicios públicos. Es así que la informalidad debe ser vista como un fenómeno complicado y multifacético. (Ramírez \& Vergara, 2014) 
Comentan Lipi \& Lipi (2017), que mientras existan impuestos, también existirá el incentivo para la evasión. Los estudios para la evasión fiscal son de interés en diferentes campos como el de la economía, las finanzas públicas, las finanzas personales, la administración de empresas, las finanzas comerciales, la contabilidad financiera, en el sistema bancario, etc. Sin embargo, existen pocos estudios sobre las causas internas y financieras. incentivos que obliguen a tomar la decisión de la evasión fiscal. (Forteza \& Noboa, 2021)

Para Saidu \& Dauda (2014), la evasión fiscal ha sido un tema de preocupación en el sistema fiscal de todos los países durante décadas. Para Muceku \& Balliu (2017), la actitud de evasión fiscal se argumenta en tener un efecto adverso en los programas socioeconómicos y políticos del gobierno. Culpando de la situación a las autoridades tributarias, por no estar a la altura de las expectativas en materia de administración tributaria; otros atribuyen el caso a la actitud antipatriótica de los contribuyentes. (Santillán \& Barbaran, 2021)

\section{ESTRATEGIAS METODOLÓGICAS O MATERIALES Y MÉTODOS}

Tipo y diseño de investigación: Investigación de tipo básica, con diseño no experimental.

\section{Esquema de diseño:}

\section{Donde:}

$\mathrm{M}=$ Muestra

$\mathrm{O} 1$ = Informalidad

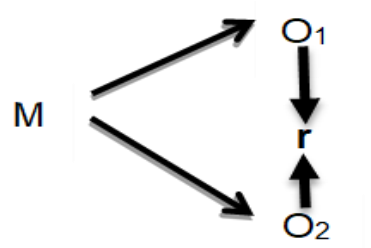

$\mathrm{O} 2$ = Evasión tributaria

$\mathrm{r}=$ Relación

Población: La población en la presente investigación estuvo constituida por un total de 40 comerciantes de abarrotes, ubicados en diferentes lugares de la localidad de Balsahuayco.

Muestra y muestreo: La muestra, teniendo en cuenta de que se trata de una población finita cuyo número no es muy grande, la muestra fue tomada como la totalidad de la población, es decir 40 comerciantes de abarrotes. El muestreo fue no probabilístico.

Técnica e instrumentos: Como técnica se ha utilizado la encuesta y como instrumento el cuestionario de encuesta, realizado para ambas variables. 
Procedimiento: El procesamiento de datos se llevó a cabo mediante: La consustanciación: Mediante la utilización de esta técnica se logró depurar los datos incensarios; Clasificación de la información: es una etapa básica en el tratamiento de los datos, se efectuó con el fin de agrupar datos por cada uno de los ítems y las dimensiones de cada una de las variables; Tabulación de datos: La información agrupada en categorías y dimensiones, después de esa tabulación se efectuó el procesamiento informático mediante programas estadísticos.

Método de análisis de datos: Se analizó los datos con lo obtenido de la aplicación de los instrumentos, se elaboró la base de datos, para facilitar el estudio de la información y avalar su posterior uso e interpretación, se utilizó el programa informático Excel para la formulación de tablas y gráficos que se requieran, se utilizó la estadística descriptiva, para realizar las tablas de frecuencias, tablas de normalidad y de relación de variables, se elaboró diagramas de barras, para la comprensión de datos más fácil, por último, para la prueba de hipótesis, se utilizó la estadística inferencial por medio de la correlación Rho de Spearman.

\section{RESULTADOS Y DISCUSIÓN}

\section{Objetivo específico 1:}

- Determinar la incidencia del desconocimiento sobre formalización en la Evasión Tributaria de los comerciantes de abarrotes de la localidad de Balsahuayco.

Tabla 1. Desconocimiento sobre formalización de los comerciantes de abarrotes de la localidad de Balsahuayco

\begin{tabular}{|l|c|c|c|c|c|c|}
\hline \multirow{2}{*}{$\begin{array}{c}\text { DIMENSIÓN: DESCONOCIMIENTO SOBRE } \\
\text { FORMALIZACIÓN }\end{array}$} & \multicolumn{2}{|c|}{ SI } & \multicolumn{2}{c|}{ NO } & \multicolumn{2}{c|}{ TOTAL } \\
\cline { 2 - 7 } & $\mathbf{N}$ & $\%$ & $\mathbf{N}$ & $\%$ & $\mathbf{N}$ & $\%$ \\
\hline $\begin{array}{l}\text { ¿Sabe usted que es la informalidad en la actividad } \\
\text { comercial que usted realiza? }\end{array}$ & 4 & 10.0 & 36 & 90.0 & 40 & 100.0 \\
\hline $\begin{array}{l}\text { ¿Sabe usted que la informalidad afecta al desarrollo } \\
\text { del país y que contribuir es parte de su deber } \\
\text { ciudadano? }\end{array}$ & 9 & 22.5 & 31 & 77.5 & 40 & 100.0 \\
\hline $\begin{array}{l}\text { ¿Conoce usted los beneficios que tiene el ser formal } \\
\text { y las sanciones para el caso de la informalidad? }\end{array}$ & 3 & 7.5 & 37 & 92.5 & 40 & 100.0 \\
\hline $\begin{array}{l}\text { ¿Ha realizado algún trámite para la creación y } \\
\text { formalización de su negocio? }\end{array}$ & 2 & 5.0 & 38 & 95.0 & 40 & 100.0 \\
\hline $\begin{array}{l}\text { ¿Usted desconoce el proceso que debe seguir para } \\
\text { formalizar su negocio? }\end{array}$ & 39 & 97.5 & 1 & 2.5 & 40 & 100.0 \\
\hline
\end{tabular}

Fuente: Elaboración propia 
Se muestra que en la dimensión desconocimiento sobre el proceso de formalización, encontramos que al ítem 1, el 90.0\% respondió NO, para el ítem 2, el 77.5\% respondieron que NO, en el ítem 3 el 92.5\% respondió que NO, en el ítem 4 el 95.0\% respondió NO, por último, en el ítem 5 el $97.5 \%$ respondió que SI. Con esto queda evidencia de que los comerciantes de abarrotes de Balsahuayco desconocen el proceso de formalización, lo que es causante de la informalidad en esta localidad.

Tabla 2. Incidencia del desconocimiento sobre el proceso de formalización en la Evasión Tributaria de los comerciantes de abarrotes de la localidad de Balsahuayco.

\begin{tabular}{|l|l|c|c|}
\hline \multicolumn{2}{|l|}{ Correlación Rho de Spearman } & $\begin{array}{c}\text { Desconocimiento } \\
\text { sobre el proceso de } \\
\text { formalización }\end{array}$ & $\begin{array}{c}\text { Evasión } \\
\text { Tributaria }\end{array}$ \\
\hline \multirow{2}{*}{$\begin{array}{l}\text { Desconocimiento } \\
\text { sobre el proceso de } \\
\text { formalización }\end{array}$} & Coeficiente de correlación & 1.000 &, $894^{* *}$ \\
\cline { 2 - 4 } & Sig. (bilateral) & 40 & 0.000 \\
\cline { 2 - 4 } & $\mathrm{N}$ &, $894^{* *}$ & 1.000 \\
\hline \multirow{2}{*}{ Evasión Tributaria } & Coeficiente de correlación & 0.000 & \\
\cline { 2 - 4 } & Sig. (bilateral) & 40 & 40 \\
\cline { 2 - 4 } & $\mathrm{N}$ & \multicolumn{2}{|c|}{} \\
\hline \multirow{2}{*}{$* *$ La correlación es significativa en el nivel 0,01 (bilateral). } \\
\hline
\end{tabular}

Fuente: Elaboración propia

Al realizar la prueba de correlación Rho de Spearman se ha obtenido una correlación positiva de 0,894 ( $\mathrm{r}=0,894)$ y un valor de $\mathrm{p}=0,000(\mathrm{p}<0,01)$, demostrándose que el desconocimiento sobre el proceso de formalización incide en la evasión tributaria de los comerciantes de abarrotes de Balsahuayco, con 99\% de confiabilidad. Es decir que mientras siga existiendo el desconocimiento sobre el proceso de formalización, existirá más evasiones tributarias por parte de los comerciantes de abarrotes

\section{Objetivo específico 2:}

- Determinar la incidencia de la ausencia de compromiso social en la Evasión Tributaria de los comerciantes de abarrotes de la localidad de Balsahuayco.

Tabla 3. Ausencia de compromiso social de los comerciantes de abarrotes de la localidad de Balsahuayco 


\begin{tabular}{|l|c|c|c|c|c|c|}
\hline \multirow{2}{*}{$\begin{array}{l}\text { DIMENSIÓN: AUSENCIA DE COMPROMISO } \\
\text { SOCIAL }\end{array}$} & \multicolumn{2}{|c|}{ SI } & \multicolumn{2}{c|}{ NO } & \multicolumn{2}{c|}{ TOTAL } \\
\cline { 3 - 8 } & $\mathbf{N}$ & $\%$ & $\mathbf{N}$ & $\%$ & $\mathbf{N}$ & $\%$ \\
\hline $\begin{array}{l}\text { ¿Considera usted que al no pagar impuestos usted } \\
\text { obtiene mayores beneficios? }\end{array}$ & 30 & 75.0 & 10 & 25.0 & 40 & 100.0 \\
\hline $\begin{array}{l}\text { ¿Si se le exigiera emitir factura, por la venta de } \\
\text { mercadería o el cliente le pidiera su comprobante de } \\
\text { pago, lo haría sin dificultad? }\end{array}$ & 4 & 10.0 & 36 & 90.0 & 40 & 100.0 \\
\hline $\begin{array}{l}\text { ¿Cuándo decide ser informal, es consiente que corre } \\
\text { el riego sobre las consecuencias de ser detectado? }\end{array}$ & 10 & 25.0 & 30 & 75.0 & 40 & 100.0 \\
\hline $\begin{array}{l}\text { ¿Realiza la declaración de sus ingresos y lleva la } \\
\text { contabilidad de su negocio? }\end{array}$ & 4 & 10.0 & 36 & 90.0 & 40 & 100.0 \\
\hline $\begin{array}{l}\text { ¿Considera usted que el comerciante debe ser } \\
\text { responsable en la declaración y en el pago de sus } \\
\text { impuestos? }\end{array}$ & 33 & 82.5 & 7 & 17.5 & 40 & 100.0 \\
\hline
\end{tabular}

Fuente: Elaboración propia

Se observa la dimensión ausencia de compromiso social, donde al ítem 6 el $75.0 \%$ respondió que SI, en el ítem 7 el 90.0\% respondieron que NO, para el ítem 8 el $75.0 \%$ respondieron que NO, en el ítem 9 el 90.0\% respondió que NO, por último, en el ítem 10 el $82.5 \%$ respondió que SI. Esto nos indica que no existe un compromiso social de parte de los comerciantes de abarrotes, otro factor que influye para que la informalidad en la localidad de Balsahuayco siga incrementando.

Tabla 4. Incidencia de la ausencia de compromiso social en la Evasión Tributaria de los comerciantes de abarrotes de la localidad de Balsahuayco

\begin{tabular}{|l|l|c|c|}
\hline \multicolumn{2}{|c|}{ Correlación Rho de Spearman } & $\begin{array}{c}\text { Ausencia de } \\
\text { compromiso }\end{array}$ & $\begin{array}{c}\text { Evasión } \\
\text { Tributaria }\end{array}$ \\
\hline \multirow{2}{*}{$\begin{array}{l}\text { Ausencia } \\
\text { compromiso }\end{array}$} & Coeficiente de correlación & 1.000 &, $873^{* *}$ \\
\cline { 2 - 4 } & Sig. (bilateral) & & 0.000 \\
\cline { 2 - 4 } & $\mathrm{N}$ & 40 & 40 \\
\hline \multirow{2}{*}{ Evasión Tributaria } & Coeficiente de correlación &, $873^{* *}$ & 1.000 \\
\cline { 2 - 4 } & Sig. (bilateral) & 0.000 & 40 \\
\cline { 2 - 4 } & $\mathrm{N}$ & 40 & \\
\hline \multirow{2}{*}{$* *$ La correlación es significativa en el nivel 0,01 (bilateral). } \\
\hline
\end{tabular}

Fuente: Elaboración propia

Al realizar la prueba de correlación Rho de Spearman se ha obtenido una correlación de $0,873(\mathrm{r}=0,873)$ y un valor de $\mathrm{p}=0,000(\mathrm{p}<0,01)$, demostrándose que la ausencia de compromiso social incide de manera negativa en la Evasión Tributaria de los comerciantes de abarrotes de la localidad de Balsahuayco, con $99 \%$ de confiabilidad. Es decir, que mientras los comerciantes no sean conscientes de lo que significa ser 
informales y evadir impuestos, se seguirá distorsionando el Sistema Tributario trayendo como consecuencia el no avance de la localidad de Balsahuayco y de todo el país.

\section{Objetivo específico 3:}

- Determinar la incidencia de los factores de informalidad en la Evasión Tributaria de los comerciantes de abarrotes de la localidad de Balsahuayco.

Tabla 5. Factores de informalidad en los comerciantes de abarrotes de la localidad de Balsahuayco

\begin{tabular}{|l|l|l|l|l|l|l|}
\hline \multirow{2}{*}{ DIMENSIÓN: Factores De Informalidad } & \multicolumn{2}{|c|}{ SI } & \multicolumn{2}{|c|}{ NO } & \multicolumn{2}{l|}{ TOTAL } \\
\cline { 2 - 9 } & $\mathbf{N}$ & $\%$ & $\mathbf{N}$ & $\%$ & $\mathbf{N}$ & $\%$ \\
\hline $\begin{array}{l}\text { ¿Considera usted que la decisión de ser informal es } \\
\text { porque el nivel de ingresos es bajo? }\end{array}$ & 25 & 62.5 & 15 & 37.5 & 40 & 100.0 \\
\hline $\begin{array}{l}\text { ¿Considera usted que los trámites para la } \\
\text { formalización son excesivos y llevan mucho } \\
\text { tiempo? }\end{array}$ & 30 & 75.0 & 10 & 25.0 & 40 & 100.0 \\
\hline $\begin{array}{l}\text { ¿Considera usted que la SUNAT no genera } \\
\text { confianza en el contribuyente? }\end{array}$ & 8 & 20.0 & 32 & 80.0 & 40 & 100.0 \\
\hline $\begin{array}{l}\text { ¿Considera usted que cuando es formal, se paga } \\
\text { más, porque las normas tributarias no son claras? }\end{array}$ & 33 & 82.5 & 7 & 17.5 & 40 & 100.0 \\
\hline $\begin{array}{l}\text { ¿Usted decide ser o continuar en la informalidad } \\
\text { porque reduce sus costos operativos en su negocio? }\end{array}$ & 30 & 75.0 & 10 & 25.0 & 40 & 100.0 \\
\hline
\end{tabular}

Fuente: Elaboración propia

Se muestra que en la dimensión factores de informalidad, al ítem 11 el 62.5\% respondió que SI, en el ítem 12 el 75.0\% respondieron que SI, para el ítem 13 el 80.0\% respondieron que NO, en el ítem 14 el 82.5\% respondió que SI, por último, en el ítem 15 el $75.0 \%$ respondió que SI. Podemos deducir que los factores que causan la informalidad en los comerciantes de abarrotes de Balsahuayco son: el nivel de ingresos, tedioso trámite de formalización, la desconfianza en la Administración tributaria, desconocimiento de normas tributarias y excesivos costos operativos.

Tabla 6. Incidencia de los factores de informalidad en la Evasión Tributaria de los comerciantes de abarrotes de la localidad de Balsahuayco

\begin{tabular}{|l|l|c|c|}
\hline \multicolumn{2}{|l|}{ Correlación Rho de Spearman } & $\begin{array}{c}\text { Factores de } \\
\text { informalidad }\end{array}$ & $\begin{array}{c}\text { Evasión } \\
\text { Tributaria }\end{array}$ \\
\hline \multirow{2}{*}{$\begin{array}{l}\text { Factores } \\
\text { informalidad }\end{array}$} & Coeficiente de correlación & 1.000 &, $886^{* *}$ \\
\cline { 2 - 4 } & Sig. (bilateral) & & 0.000 \\
\cline { 2 - 4 } & $\mathrm{N}$ & 40 & 40 \\
\hline \multirow{2}{*}{ Evasión Tributaria } & Coeficiente de correlación &, $886^{* *}$ & 1.000 \\
\cline { 2 - 4 } & Sig. (bilateral) & 0.000 & \\
\hline
\end{tabular}


\begin{tabular}{|l|l|}
$\mid \mathrm{N}$ & 40 \\
\hline$* *$. La correlación es significativa en el nivel 0,01 (bilateral). & 40 \\
\hline
\end{tabular}

Fuente: Elaboración propia

Al realizar la prueba de correlación Rho de Spearman se ha obtenido una correlación de $0,886(\mathrm{r}=0,886)$ y un valor de $\mathrm{p}=0,000(\mathrm{p}<0,01)$, demostrándose que los factores de informalidad inciden de manera negativa en la Evasión Tributaria de los comerciantes de abarrotes de la localidad de Balsahuayco, con 99\% de confiabilidad. Es decir, los factores de informalidad (el nivel de ingresos, tedioso trámite de formalización, la desconfianza en la Administración tributaria, desconocimiento de normas tributarias y excesivos costos operativos), hacen que la evasión tributaria siga aumentando, siendo perjudicial para el desarrollo del país.

\section{Objetivo General:}

- Determinar la incidencia de la Informalidad en la Evasión Tributaria de los comerciantes de abarrotes de la localidad de Balsahuayco - Jaén, primer semestre 2021.

Tabla 7. Informalidad de los comerciantes de abarrotes en la localidad de Blasahuayco

\begin{tabular}{|l|c|c|}
\hline Informalidad & f & \% \\
\hline $\mathrm{Si}$ & 40 & 100.0 \\
\hline No & 0 & 0.0 \\
\hline Total & $\mathbf{4 0}$ & $\mathbf{1 0 0 . 0}$ \\
\hline
\end{tabular}

Fuente: Elaboración propia

Se muestra que el $100.0 \%$ de los comerciantes de la localidad de Balsahuayco son informales, teniendo en cuenta las tablas anteriores se deduce que esto se debe al desconocimiento sobre la formalización y sus beneficios, a la falta de compromiso y responsabilidad social con el país y a diversos factores que influyen para que los comerciantes decidan voluntariamente ser informales.

Tabla 8. Incidencia de la informalidad en la Evasión tributaria de los comerciantes de abarrotes de la localidad de Balsahuayco.

\begin{tabular}{|l|l|c|c|}
\hline \multicolumn{2}{|l|}{ Correlación Rho de Spearman } & Informalidad & $\begin{array}{c}\text { Evasión } \\
\text { Tributaria }\end{array}$ \\
\hline \multirow{4}{*}{ Informalidad } & Coeficiente de correlación & 1.000 &, $855^{* *}$ \\
\cline { 2 - 4 } & Sig. (bilateral) & & 0.000 \\
\cline { 2 - 4 } & $\mathrm{N}$ & 40 & 40 \\
\hline
\end{tabular}




\begin{tabular}{|l|l|c|c|}
\multirow{4}{*}{ Evasión Tributaria } & Coeficiente de correlación &, $855^{* *}$ & 1.000 \\
\cline { 2 - 4 } & Sig. (bilateral) & 0.000 & 40 \\
\cline { 2 - 3 } & $\mathrm{N}$ & 40 & \\
\hline **. La correlación es significativa en el nivel 0,01 (bilateral). \\
\hline \multicolumn{2}{|c|}{ Fuente: Elaboración propia }
\end{tabular}

Con la prueba de correlación Rho de Spearman se obtuvo una correlación de 0,855 (r= $0,855)$ y un valor de $\mathrm{p}=0,000(\mathrm{p}<0,01)$, demostrándose que existe incidencia de la Informalidad en la Evasión Tributaria de los comerciantes de abarrotes de la localidad de Balsahuayco. Es decir, que a mayor informalidad mayor incremento en la evasión tributaria. Aceptando la hipótesis general de la investigación, rechazando la hipótesis nula.

Con estos resultados se pasa a discutir con las investigaciones estudiadas, respecto al primer objetivo específico se encontró que el $90.0 \%$ no conoce el concepto de informalidad, el $77.5 \%$ desconoce que la informalidad afecta el desarrollo del país, el 92.5\% no conoce los beneficios de la formalización y las consecuencias de la informalidad, el 95.0\% no ha realizado ningún trámite de formalización, además el 97.5\% no conoce el proceso de formalización, deduciendo que la gran mayoría de los informales desconocen lo que significa ser formales, esto conlleva a que cometan evasiones tributarias, además se obtuvo una correlación Rho de Spearman de 0,894 demostrándose que existe incidencia del desconocimiento del proceso de formalización en la Evasión Tributaria de los comerciantes de abarrotes de la localidad de Balsahuayco, lo que permitió aceptar la primera hipótesis específica de la investigación, es decir el desconocimiento sobre formalización incide negativamente en la Evasión Tributaria de los comerciantes de abarrotes, en otras palabras el no conocer ni estar informado de la importancia de la formalización para nuestro país está provocando que exista más evasiones tributarias que afectan de forma significativa la hacienda pública, provocando que el país no se desarrolle debido a que solo hay recursos para cubrir las necesidades básicas de la población. Concordando con Fossa (2017), quien encontró que la mayoría de informales, lo son porque no saben el proceso de formalización, ósea no conocen los trámites, licencias y otros documentos para empezar con buen pie los negocios. Entonces ellos no saben de los beneficios que tiene el ser formal y las sanciones que trae consigo la informalidad. 
De acuerdo al segundo objetivo específico se encontró que el $75.0 \%$ considera que al no pagar impuestos se obtiene mayores beneficios, el $90.0 \%$ no emiten comprobantes de pago por sus ventas, el $75.0 \%$ decide ser informal sin importarles las consecuencias al ser detectado, el $90.0 \%$ no declara sus ingresos ni tampoco lleva la contabilidad de su negocio, esto indica que la mayoría de los comerciantes no quieren ser responsables socialmente de los tributos, protegiendo su propia economía sin importarles el país, ello conlleva al incremento de evasiones en la localidad de Balsahuayco, además se obtuvo una correlación Rho de Spearman de 0,873 demostrándose que existe incidencia de la ausencia de compromiso social en la Evasión Tributaria de los comerciantes de abarrotes de la localidad de Balsahuayco, lo que permitió aceptar la segunda hipótesis específica de la investigación, demostrándose que la ausencia de compromiso social incide de manera negativa en la Evasión Tributaria de los comerciantes de abarrotes, es decir, que mientras los comerciantes no sean conscientes de lo que significa ser informales y evadir impuestos, se seguirá distorsionando el Sistema Tributario trayendo como consecuencia el no avance de la localidad de Balsahuayco y de todo el país. Estando de acuerdo con Fossa (2017), quien encontró que los informales no tienen compromiso para el cumplimiento del pago de tributos, debido a que no conocen ni sus derechos, ni el deber de ciudadanos de financiar al estado con la tributación. Para Cubas (2017), la insensibilización de las jurisdicciones y la terquedad de los informales crean discrepancia en ambos fragmentos por lo que siempre habrá un desacuerdo recíproco, así las acciones de los informales tendrán un efecto perjudicial en el cobro de tributos del país.

En el tercer objetivo específico se encontró que el $62.5 \%$ considera ser informal porque el nivel de ingresos es bajo, el $75.0 \%$ considera que los trámites para la formalización son excesivos y demandan mucho tiempo, el $80.0 \%$ no considera que la SUNAT de confianza al contribuyente, el $82.5 \%$ considera que el ser formal conduce a un pago excesivo debido a que las normas tributarias no son claras, y el $75.0 \%$ decide ser informal porque reduce los costos operativos en su negocio, con ello se evidencia que los comerciantes tienen muchos factores que los incitan a ser informales sin importarles caer en evasiones tributarias que son castigadas por la Administración tributaria, ya que muestran rechazo a la idea de que el Estado administre los recursos de la población, por falta de perspectiva al futuro e insuficientes expectativas como dueños del negocio, además se obtuvo una correlación Rho de Spearman de 0,886 demostrándose que existe incidencia de los 
factores de informalidad en la Evasión Tributaria de los comerciantes de abarrotes de la localidad de Balsahuayco, lo que permitió aceptar la tercera hipótesis específica de la investigación, demostrándose que los factores de informalidad inciden de manera negativa en la Evasión Tributaria de los comerciantes de abarrotes, es decir que el nivel de ingresos, los trámites tediosos de formalización, la desconfianza en la Administración tributaria, desconocimiento de normas tributarias y excesivos costos operativos, hacen que la evasión tributaria siga aumentando, siendo perjudicial para el desarrollo del país. Quispe (2016), encontró que existen tres factores que aumentan la informalidad; los impuestos por trabajadores, pésimo marco legal en seguridad nacional, políticas económicas que inquietan a las secciones más prósperos en elegir la informalidad y reformas comerciales que están hechas sin tener el impacto que podrían causar en las secciones de menor productividad.

De acuerdo al objetivo general, se obtuvo como resultados que el $100.0 \%$ de los comerciantes de abarrotes de la localidad de Balsahuayco son informales, ocasionando así las evasiones tributarias en dicha localidad, además se obtuvo una correlación Rho de Spearman de 0,855 demostrándose que existe incidencia de la Informalidad en la Evasión Tributaria de los comerciantes de abarrotes de la localidad de Balsahuayco, lo que permitió aceptar la hipótesis general de la investigación, es decir que la informalidad incide de manera negativa en la evasión tributaria, en otras palabras a mayor informalidad mayor incremento de la evasión tributaria, siendo perjudicial para la nación, porque no permite el progreso y desarrollo del país. Kanbur \& Keen (2015), explican que la informalidad mayormente va entendida como la no remisión de la cantidad total de impuestos adeudados o falta de pago, pero las razones van más allá del no pago de impuestos, como por ejemplo algunos no tienen los ingresos suficientes para que legalmente estén obligados a pagar impuestos, en algunos casos porque redujeron su actividad para llegar por debajo de ese límite, o podrían estar evadiendo, sin pagar de manera deshonesta, deduciendo que el motivo por el que una empresa o un individuo no paga impuestos puede importar tanto o más que el hecho de que no pagan nada. Al respecto Quispe (2016), encontró que la informalidad se encuentra mayormente en personas, microempresas y pequeñas empresas debido a las actividades que estas desarrollan, provocando un desequilibrio social, contratando también personal de manera informal, lo que no les permite acceder legalmente a sus derechos de salud y laborales. 
Por otro lado, Proaño (2015) determina que los países quieren conseguir tácticas legales que puedan mejorar la cultura tributaria, dando beneficios para conseguir buenos contribuyentes, según el estudio durante los tres últimos años ha existido un crecimiento en la recaudación, dando a entender que muchos contribuyentes decidieron ser formales; aparte también se logró determinar la diferencia entre el régimen general y el régimen simplificado, esto es, que el régimen formal va dirigido a personas naturales y empresas constituidas, mientras que el régimen simplificado para las acciones que no son formales que tienen determinado la categoría para sus oportunas cuotas a pagar, intuyendo que los que pasan a ser formales prefieren el régimen simplificado. Para Mendiburu (2016), la informalidad se da debido a los costos excesivos de formalización en algunos países, por los procesos de inscripción y registro, también para ingresar a sectores grandes y complicados, así como para quedar dentro del mismo, pago de tributos, cumplir las leyes laborales, manejo ambiental, salud, entre otros. Por otro lado, Ordoñez \& Tenesaca (2014), señalan que existen beneficios por ser formales, tales como la defensa policial frente al crimen y el abuso, la intervención de la ley judicial para resolver conflictos y el acatamiento de contratos en su totalidad, beneficios en instituciones financieras formales que entreguen créditos minimizando riesgos, y por último posibilita la expansión del negocio a mercados locales e internacionales.

Fortaleciendo la presente investigación se ha tomado en cuenta los estudios realizados por otros autores, con respecto a la informalidad Bu \& Cuervo (2020), encontraron que los costos de informalidad quedan impresos y afectan las relaciones con empleados, proveedores y distribuidores, limitando los incentivos de las empresas y su capacidad para innovar incluso después de la formalización, es por ello que las empresas creadas informalmente se involucran más en la imitación y menos en el desarrollo de nuevos productos innovadores. Mientras que Ulyssea (2020), dice que no podemos considerar la reducción de los costos de la formalidad como una política eficaz para reducir la informalidad, aunque esto tenga efectos agregados positivos, como una mayor producción y productividad total de los factores, sino que la política de formalización más eficaz es aumentar la aplicación en el margen extensivo, pero no en el margen intensivo de la informalidad. Con respecto a evasión tributaria Paredes (2017), encontró que esta se genera por la ineficiente redistribución de lo recaudado, las suspensiones socio económico en los comercios, es por ello que este sector se encuentra desinformado y no 
existe un cumplimiento a gran escala, con esto podemos deducir que las políticas fiscales no son están claras para el público en general, trayendo como consecuencia el incumplimiento de tributos y la poca recaudación fiscal en los sectores informales del sector comercio. Para Mawejje \& Okumu (2016), el alcance de la evasión fiscal está asociado con el soborno burocrático, la calidad y eficiencia de los sistemas legales y la provisión inadecuada de capital público.

\section{CONCLUSIÓN O CONSIDERACIONES FINALES}

De acuerdo al objetivo principal de la investigación se encontró que el 100.0\% de los comerciantes de abarrotes de la localidad de Balsahuayco son informales, ocasionando así las evasiones tributarias en dicha localidad, determinándose que la informalidad incide de manera negativa en la evasión tributaria, datos demostrados mediante una correlación Rho de Spearman de 0,855 y un nivel de significancia de 0,01 .

Por otro lado, se encontró que el $90.0 \%$ desconoce el concepto de informalidad, el $77.5 \%$ desconoce que la informalidad afecta el desarrollo del país, el 92.5\% no conoce los beneficios de la formalización y las consecuencias de la informalidad, el 95.0\% no ha realizado ningún trámite de formalización, además el $97.5 \%$ no conoce el proceso de formalización, esto conlleva a que el 100.0\% cometa evasiones tributarias, determinándose que el desconocimiento sobre formalización incide de manera negativa en la evasión tributaria de los comerciantes de abarrotes de la localidad de Balsahuayco, datos demostrados con una correlación Rho de Spearman r=0,894 y un valor de significancia 0,01 .

También se encontró que el $75.0 \%$ considera que al no pagar impuestos se obtiene mayores beneficios, el $90.0 \%$ no emiten comprobantes de pago por sus ventas, el $75.0 \%$ decide ser informal sin importarles las consecuencias al ser detectado, el 90.0\% no declara sus ingresos ni tampoco lleva la contabilidad de su negocio, haciendo que el $100.0 \%$ cometa evasiones tributarias, determinándose que la ausencia de compromiso social incide de manera negativa en la Evasión Tributaria de los comerciantes de abarrotes de la localidad de Balsahuayco, demostrado con una correlación Rho de Spearman r= 0,873 y un valor de significancia de 0,01 .

Por último, se encontró que el $62.5 \%$ considera ser informal porque el nivel de ingresos es bajo, el $75.0 \%$ considera que los trámites para la formalización son excesivos y demandan mucho tiempo, el $80.0 \%$ no considera que la SUNAT de confianza al 
contribuyente, el $82.5 \%$ considera que el ser formal conduce a un pago excesivo debido a que las normas tributarias no son claras, y el $75.0 \%$ decide ser informal porque reduce los costos operativos en su negocio, todo ello conlleva a evasiones tributarias en un $100.0 \%$, determinándose que los factores de informalidad inciden negativamente en la evasión tributaria de los comerciantes de abarrotes de la localidad de Balsahuayco, datos contrastados con correlación Rho de Spearman r=0,886 y un valor de significancia 0,01 .

\section{LISTA DE REFERENCIAS}

Adriano Pérez, C. (2018). Documento de trabajo definiciones y repercusiones de la informalidad. Centro de Investigación de economía y negocios globales, 16.

Alcalde Huanca, E. (2019). Evasión Tributaria y su Incidencia en la Recaudación del Impuesto Predial en la Municipalidad Provincial de Jaén. Tesis de grado, Universidad César Vallejo, Chiclayo.

Bu, J., \& Cuervo Cazurra, A. (14 de julio de 2020). Informality costs: Informal entrepreneurship and innovation in emerging economies. Strategic Entrepreneurship Journal, 14(3), 329-368.

Chávez Macías, A., Peña Cárdenas, M., \& Velazco Sandoval, N. (2015). Informal trade, strategies aimedat tax reduction. Global Conference on Business and Finance Proceedings, 10(1).

Collosa, A. (22 de Abril de 2019). Evasión Tributaria en América Latina: Un Llamado de atención para todos. Obtenido de Centro Interamericano de Administraciones Tributarias: https://www.ciat.org/evasion-tributaria-en-america-latina-un-llamadode-atencion-para-todos/

Cubas Quiroz, U. N. (2017). Efectos de la informalidad de las Mypes en la recaudación tributaria del distrito de José Leonardo Ortiz de la provincia de Chiclayo, año 2016 -2017 .

Dienes, A. (15 de diciembre de 2018). Informality trap: a foundation of Russia's statistpatrimonial capitalism. Contemporary Politics, 25, 334-352.

Forteza, A., \& Noboa, C. (6 de July de 2021). Tolerance of tax evasion. International Economics and Economic Policy.

Fossa Crespo, G. A. (2017). Análisis de la Informalidad y Evasión Tributaria en los hospedajes ubicados en la urbanización Piura, 2017. 
Hernández, M., \& De la Roca, J. (2016). Evasión tributaria e informalidad en el Perú. Economía y Sociedad, 62.

Kanbur, R., \& Keen, M. (27 de Febrary de 2015). Reducing Informality: It may be a great slogan, but it is of little value as a practical objective for tax reform. Finance \& Development, 52(1), 1-60. Recuperado el 25 de Julio de 2021, de https://doi.org/10.5089/9781498351942.022

Lavado, P. (25 de noviembre de 2016). Informalidad de Perú. Obtenido de Foco Económico un blog latinoamericano de economía y política: http://focoeconomico.org/2016/11/25/informalidad-en-peru/

Lipi, I., \& Lipi, R. (2017). Small Business Tax Evasion, is There a Case of Internal Finance? Journal: Acta Universitatis Danubius. Económica, 45-54.

Luján, R., \& Cano, E. (2019). La Cultura Tributaria y el Comportamiento de los Delitos Tributarios en el Perú: Periodo 2012-2016. Revista Ciencia y Tecnología, 15(2).

Mawejje, J., \& Okumu, I. (21 de September de 2016). Tax Evasion and the Business Environment in Uganda. South African Journal of Economics, 84(3). Obtenido de https://onlinelibrary.wiley.com/doi/epdf/10.1111/saje.12132

Mendiburu Rojas, A. O. (2016). Factores que propician la informalidad de las pymes y su incidencia en el desarrollo de estas en la provincia de Trujillo, Distrito de Victor Larco. Tesis de grado, Universidad Nacional de Trujillo, Trujillo.

Muceku, H., \& Balliu, T. (07 de enero de 2017). The Albanian Taxpayers' Perception of the Current Tax System and Its Impact on Informal Economy, Tax Evasion and Tax Avoidance. Mediterranean Journal of Social Sciences, 8(1).

Ordoñez, R., \& Tenesaca, M. (2014). La cultura tributaria desde la informalidad de los comerciantes de la Bahía de Guayaquil. Yachana (Revista Científica), 3(2). Recuperado el 10 de Julio de 2021, de https://doi.org/10.1234/ych.v3i2.20

Paredes Ramos, M. P. (2017). La evasión tributaria y la recaudación generada por los comerciantes informales de ropa del Mercado Mayorista de la ciudad de Ambato. Tesis, Universidad Técnica de Ambato, Ambato.

Proaño Males, E. (2015). La obligación tributaria en el comercio informal ecuatoriano mediante la aplicación del régimen impositivo simplificado ecuatoriano - RISE. Universidad Central del Ecuador, Quito. Obtenido de http://www.dspace.uce.edu.ec/bitstream/25000/5163/1/T-UCE-0013-Ab-340.pdf 
Quispe Delgado, D. (2016). La informalidad y su relación con la evasión tributaria en los comerciantes del distrito de Anco Huallo. Tesis, Universidad José Carlos Mariátegui, Moquegua.

Quispe Pérez, N. (2020). La informalidad en la evasión tributaria de las MYPES de los comerciantes del mercado internacional san José rubro venta de prendas de vestir Juliaca, 2019. Tesis de grado, Universidad Católica Los Ángeles de Chimbote, Juliaca.

Ramírez Reyes, J. C., \& Vergara Mesa, G. A. (2014). Revista Científica Guillermo de Ockham. Oportunidades, sostenibilidad y riesgos de los negocios informales de la comuna 10 de Cali, 12(1), 35-41.

Rodríguez Soto, J., \& Dussán Pulecio, C. (2018). La informalidad empresarial, evolución literaria que denota un fenómeno complejo. Polo del Conocimiento.

Saidu, S., \& Dauda, U. (2014). Tax Evasion and Governance Challeneges in the Nigerian Informal Sector. Journal of Finance and Economics, 2(5), 156-161.

Santillán Ríos, C., \& Barbaran Mozo, P. (2021). La figura de la evasión tributaria y sus implicancias en el desarrollo socioeconómico del estado. Ciencia Latina Revista Multidisciplinar, 5(4).

Ulyssea, G. (1 de May de 2020). Informality: Causes and consequences for development. Annual Review of Economics, 12, 525-546.

Verona, J. (27 de marzo de 2019). El sector informal y la evasión tributaria. Obtenido de Grupo Verona: https://grupoverona.pe/el-sector-informal-y-la-evasion-tributaria/ 